\title{
Rotational Atherectomy and Stent Implantation for Calcified Left Main Lesions
}

\author{
Bryan G. Schwartz ${ }^{\mathrm{a}, \mathrm{d}}$, Guy S. Mayeda ${ }^{\mathrm{b}}$, Christina Economides ${ }^{\mathrm{b}}$, Robert A. Kloner ${ }^{\mathrm{a}, \mathrm{c}}$, \\ David M. Shavelle ${ }^{c}$, Steven Burstein ${ }^{\mathrm{b}}$
}

\begin{abstract}
Background: Left main coronary artery (LMCA) bifurcation and heavily calcified lesions are common and challenging to treat percutaneously. Rotational atherectomy (RA) may be beneficial in this setting to facilitate stent placement though direct supporting evidence is lacking. This study sought to analyze patients who underwent RA of the LMCA.
\end{abstract}

Methods: Consecutive cases involving RA of the LMCA between $1 / 1 / 2004$ and $12 / 31 / 2009$ at a private, tertiary referral hospital were reviewed retrospectively. Medical records, angiograms and clinically driven follow-up were reviewed.

Results: Thirty-one cases were identified (20 protected, 11 unprotected), including 23 with stent implantation (21 drug-eluting, 2 bare metal). All 31 lesions had moderate to severe calcification, $84 \%$ involved the distal segment. Mean burr-to-vessel ratio was 0.43 . Overall angiographic success was $90 \%(28 / 31)$ and was higher with a drug-eluting stent versus no stent $(100 \%$ vs. $62 \% ; \mathrm{P}=$ 0.0153). In-hospital major adverse cardiovascular events (MACE) occurred in 1 patient (3\%). Mid-term MACE occurred in 6 patients $(26 \%)$ and tended to occur less frequently in patients with protected LMCAs $(\mathrm{P}=0.0697)$. At final follow-up, patients were more likely to be alive and free from angina with a protected LMCA (94\% vs. $57 \%$ unprotected; $\mathrm{P}=0.0564)$ and with a drug-eluting stent $(89 \%$ vs. $50 \%$ with no stent; $\mathrm{P}=0.0281$ ).

Manuscript accepted for publication August 17, 2011

${ }^{a}$ Heart Institute, Good Samaritan Hospital, Los Angeles, California, USA

${ }^{\mathrm{b}}$ Department of Cardiology, Good Samaritan Hospital, Los Angeles, California, USA

${ }^{\mathrm{c}}$ Department of Internal Medicine, Division of Cardiovascular Medicine, Keck School of Medicine at the University of Southern California, Los Angeles, CA, USA

${ }^{\mathrm{d} C}$ Corresponding author: Bryan Schwartz, Heart Institute, Good Samaritan Hospital, 1225 Wilshire Blvd., Los Angeles, CA 90017-2395, USA. Email: bschwartz15@hotmail.com

doi: $10.4021 / \mathrm{cr} 78 \mathrm{w}$
Conclusions: RA of the LMCA to facilitate stent implantation appears to be safe and effective with favorable mid-term outcomes. In the setting of severe calcification and distal LMCA involvement RA and drug-eluting stent implantation should be considered.

Keywords: Cardiac catheterization; Drug-eluting stent; Devices

\section{Introduction}

Percutaneous coronary intervention (PCI) is often performed on patients with significant disease in a protected (left coronary grafted) left main coronary artery (LMCA). For selected patients with unprotected LMCA disease, recent evidence regarding PCI with drug-eluting stent (DES) implantation suggests a benefit similar to coronary artery bypass graft (CABG) surgery, although this approach is highly debated $[1,2]$. For patients with unprotected LMCA disease who are not eligible for CABG, PCI currently has a class II indication [3]. Certain LMCA lesion subsets remain especially challenging for PCI, including the distal bifurcation and heavily calcified lesions $[2,4,5]$. The distal bifurcation is involved in over $60 \%$ of unprotected LMCA lesions and predicts a worse clinical outcome $[2,4,5]$. Alternative interventions, such as the use of debulking devices, may improve the endovascular management of these lesion subsets.

Rotational atherectomy (RA) utilizes a diamond coated burr that rotates at high speed $(120,000-200,000$ revolutions per minute) to ablate atherosclerotic plaque [6]. In heavily calcified lesions, RA facilitates stent implantation and improves acute gain [7-11]. Also, RA preserves the patency of side branches in bifurcation and ostial lesions [12, 13]. RA alone or with bare metal stents resulted in unacceptably high restenosis rates; however, at 6 months to 3 years of followup RA with DESs resulted in target lesion revascularization rates ranging from $2 \%$ to $10.6 \%$ [14-18]. Reports of RA involving the LMCA are scarce [19]. We sought to analyze procedural characteristics and clinical outcomes in a group of patients that underwent RA to facilitate DES implantation in the LMCA to better define the use of RA in the LMCA in 
contemporary practice.

\section{Methods}

Approval was obtained from the Western Institutional Review Board. For this retrospective analysis, the cardiac catheterization database at a private, tertiary referral hospital was searched to identify all cases involving RA in the LMCA between 1/1/2004 and 12/31/2009. A comprehensive chart review was conducted to record pertinent data on each case, including demographics, medical history, procedural characteristics, hospital course, and mid-term follow-up. Angiograms were reviewed and quantitative coronary angiography (QCA) was performed to define lesion and interventional characteristics.

\section{Definitions}

Lesion calcification was defined prior to contrast injection as follows: severe if radiopacities were readily apparent without cardiac motion, moderate if radiopacities were apparent only with cardiac motion, mild if faint radiopacities were seen only with cardiac motion, or none if no radiopacities were apparent [20]. A lesion was considered bifurcating if a branch $(>1.5 \mathrm{~mm})$ originated within the stenosis, the branch had ostial disease, and the branch was completely surrounded by stenotic portions within the parent vessel lesion [20]. Clinical and angiographic follow-up were clinically driven, not mandated. Clinical follow-up was only included for patients that returned to our facility and were evaluated by our physicians. Major adverse cardiac events (MACE) were defined as death, Q-wave myocardial infarction, target vessel revascularization, or new creatine kinase elevation above 2 times the upper limit of normal post-PCI. Angiographic success was defined as $<40 \%$ residual stenosis and TIMI grade 3 flow at the conclusion of the procedure. Procedural success was defined as angiographic success in the absence of MACE. Target vessel revascularization was defined as PCI or CABG to treat restenosis within the LMCA. Complications occurring during the index hospitalization were classified as acute, and complications occurring after hospital discharge were considered mid-term (mean 11.4 months; range 0 - 54 months). Binary restenosis was defined as $>50 \%$ diameter stenosis of the target lesion at follow-up angiography.

\section{Treatment}

All medical decisions, including medications and PCI, were at the discretion of the interventionalist. RA was used primarily to change the compliance of a calcified artery (30 of $31,97 \%$ ) and to prevent side branch occlusion (16 of 31 , $52 \%$ ) (Table 1). In 2 patients (6\%) RA was not planned but was used after attempts to deliver a balloon had failed. The final burr-to-vessel ratio was $0.43 \pm 0.07$.

Balloon angioplasty was used in 26 cases (84\%) with a mean maximum inflation pressure of $9.1 \pm 4$.9atm. Stents were implanted in 23 lesions (81\%), 21 of which were DESs (12 Taxus, Boston Scientific, Natick, MA, USA; 6 Cypher, Cordis, Bridgewater, NJ, USA; 3 other). The 2 bare metal stents (Multi-Link Vision, Abbott Vascular, Abbott Park, IL, USA) were used because of a history of stent thrombosis in 1 case, and impending surgery for bladder cancer in another. Stents were not used in 8 cases due to vessel size mismatch ( 3 patients), a good result after RA (3 patients) and to avoid jailing a major branch (2 patients). In this institution, when DESs are implanted in an unprotected LMCA, clopidogrel is continued indefinitely unless the risk of bleeding becomes too high.

Procedure times were long (mean $99 \pm 24 \mathrm{~min}$ ) and contrast use was high (mean $208 \pm 86 \mathrm{ml}$ ). Utilization of intravascular ultrasound was low $(4 / 31 ; 13 \%)$. Eight of 11 (73\%) procedures on unprotected LMCAs were performed with a prophylactic left ventricular assist device (in 7 cases TandemHeart ${ }^{\mathrm{TM}}$, Cardiac Assist, Inc, Pittsburgh, PA, USA). All patients treated with prophylactic left ventricular assist devices had an ejection fraction $\leq 40 \%$ (mean $28 \pm 10 \%$ ).

\section{Quantitative coronary angiography}

QCA was performed by an experienced angiographer using MDQM-QCA (Medcon Quantitative Measurements - Quantitative Coronary Arteriography, Medcon Limited, Tel Aviv, Israel) edge-detection software. For each image calibration was done by performing QCA on a segment of catheter with known diameter. All QCA measurements were confined to the LMCA only, even if the intervention also involved another coronary artery (i.e. ostial circumflex). The images with the least amount of foreshortening and the highest degree of stenosis were selected for analysis. Lesion length was determined using the pre-intervention angiogram with the least amount of foreshortening. Reference vessel diameter was determined using the final image (post-intervention) at the angiographically normal-appearing proximal LMCA (for proximal lesions the segment with the largest diameter was used). Minimal luminal diameter (MLD) was determined up to four times: 1) pre-intervention, 2) post-RA (and balloon angioplasty if balloon angioplasty was done), 3) post-stent (if a stent was placed), and 4) at follow-up (if available). The \% diameter stenosis at each instance was determined by dividing the MLD at that instance by the reference vessel diameter. Acute gain was defined as post-intervention MLD minus pre-intervention MLD. Late loss was defined as postintervention MLD minus follow-up MLD.

\section{Statistics}

Results are reported as the mean \pm standard deviation or per- 


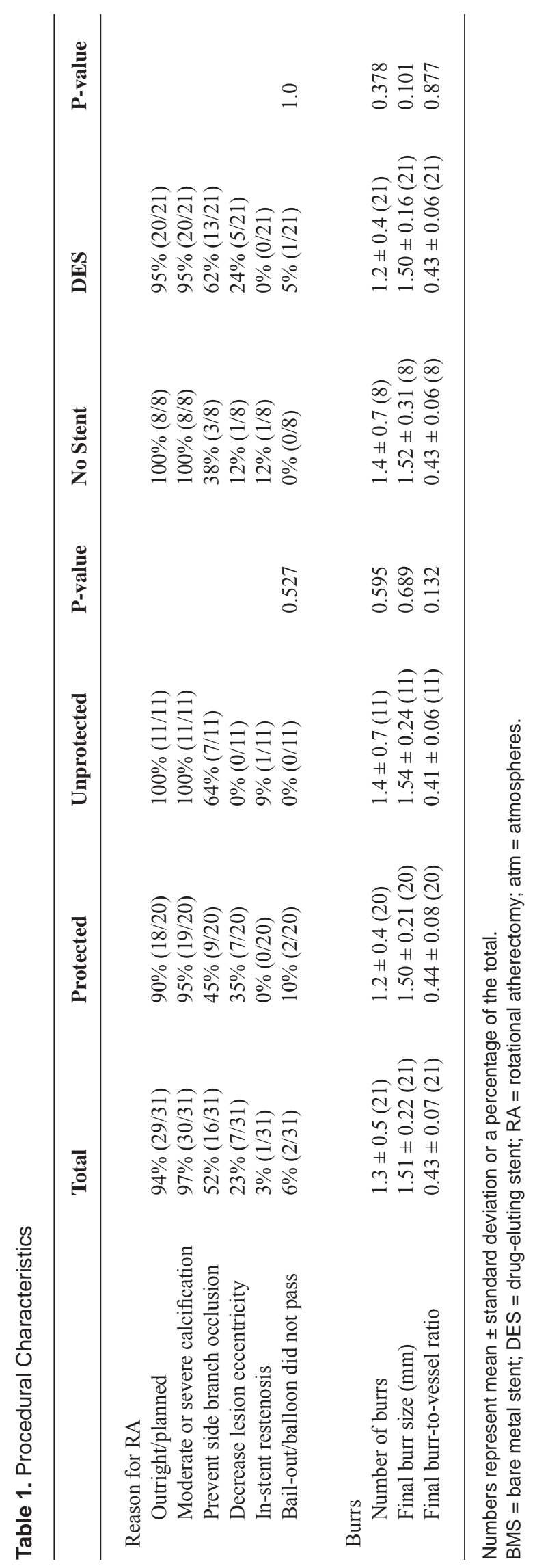

centages of the total. Patients with protected LMCAs were compared with patients with unprotected LMCAs. Patients treated with a DES were compared with patients treated without a stent (patients treated with a BMS were excluded from this comparison). Student's t-test was used to compare continuous variables. Fischer's exact test was used to compare class variables (SAS 9.1, Cary, NC). Kaplan-Meier estimates and log-rank tests were performed using the $\mathrm{R}$ statistical software system, Ver:2.11.1 (www.r-project.org). Statistical significance was considered a P-value $<0.05$.

\section{Results}

A total of 31 patients underwent RA of the LMCA, including 20 with protected and 11 with unprotected lesions (Table 2). Twenty-one patients received a DES, 2 received a bare metal stent and 8 patients did not receive a stent. Patients were elderly (mean $75.5 \pm 9.4$ years) with longstanding coronary artery disease $(68 \%$ had a history of CABG at a mean of 10.0 years prior). Medical comorbidities included hypertension in $84 \%$, hyperlipidemia in $65 \%$ and diabetes mellitus in $61 \%$. In addition to left main disease, 26 of 31 patients $(84 \%)$ also had 3 vessel coronary disease and many patients had severe comorbidities and poor functional status. Baseline characteristics were similar between groups, except that patients undergoing unprotected LMCA interventions had lower ejection fractions than those with protected LMCAs. The indications for PCI of the LMCA in the 11 patients with unprotected disease included severe comorbidities (5 patients), poor functional status (5 patients), advanced age (4 patients), and poor targets (2 patients) (some patients had multiple reasons). Patients undergoing interventions on protected LMCAs tended to present with angina, whereas a recent myocardial infarction was more common in patients with unprotected LMCAs.

All LMCA lesions were moderately (3 lesions; 10\%) or severely (28 lesions; 90\%) calcified. Most lesions involved the distal segment of the LMCA (26 of 31; 84\%) and the bifurcation ( 20 of $31 ; 65 \%$ ) (some distal LMCA lesions with totally occluded branches were not bifurcation lesions). Interventions on protected LMCAs more often involved the ostial left circumflex coronary artery (14 of 20 patients, $70 \%$ ). Conversely, interventions on unprotected LMCAs tended to involve the ostial left anterior descending coronary artery (7 of 11 patients, $64 \%$ ).

Angiographic success was achieved in 28 of the 31 patients $(90 \%)$, and procedural success in 27 of $31(87 \%)$ (Table 3). In patients without a reason to avoid a DES, RA successfully facilitated DES implantation in all cases (21 of 21 patients, $100 \%$ ) despite severe calcification. All PCIs with DES implantation achieved angiographic success (21 of $21 ; 100 \%)$. Angiographic success was significantly more likely in patients treated with a DES compared with no stent 


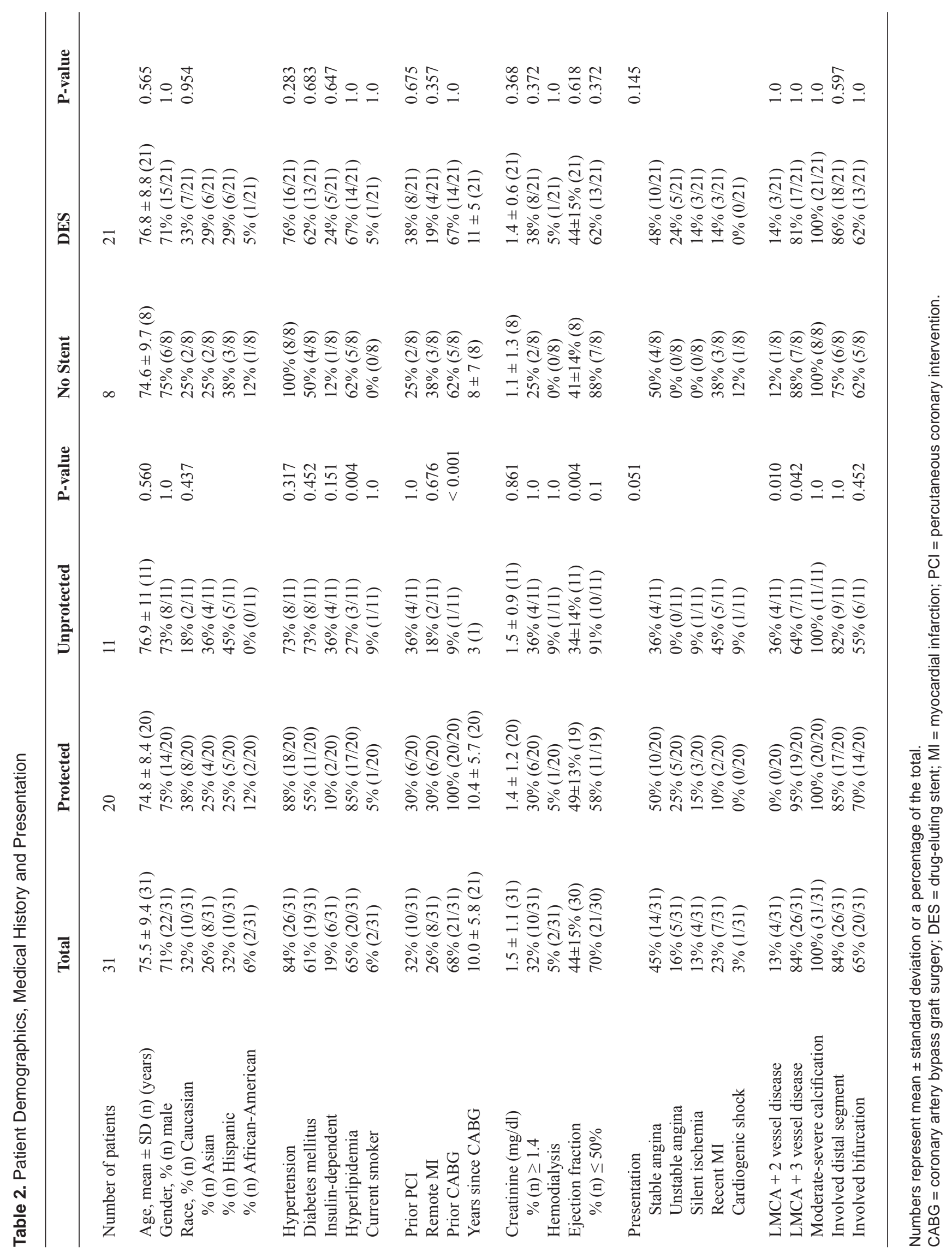




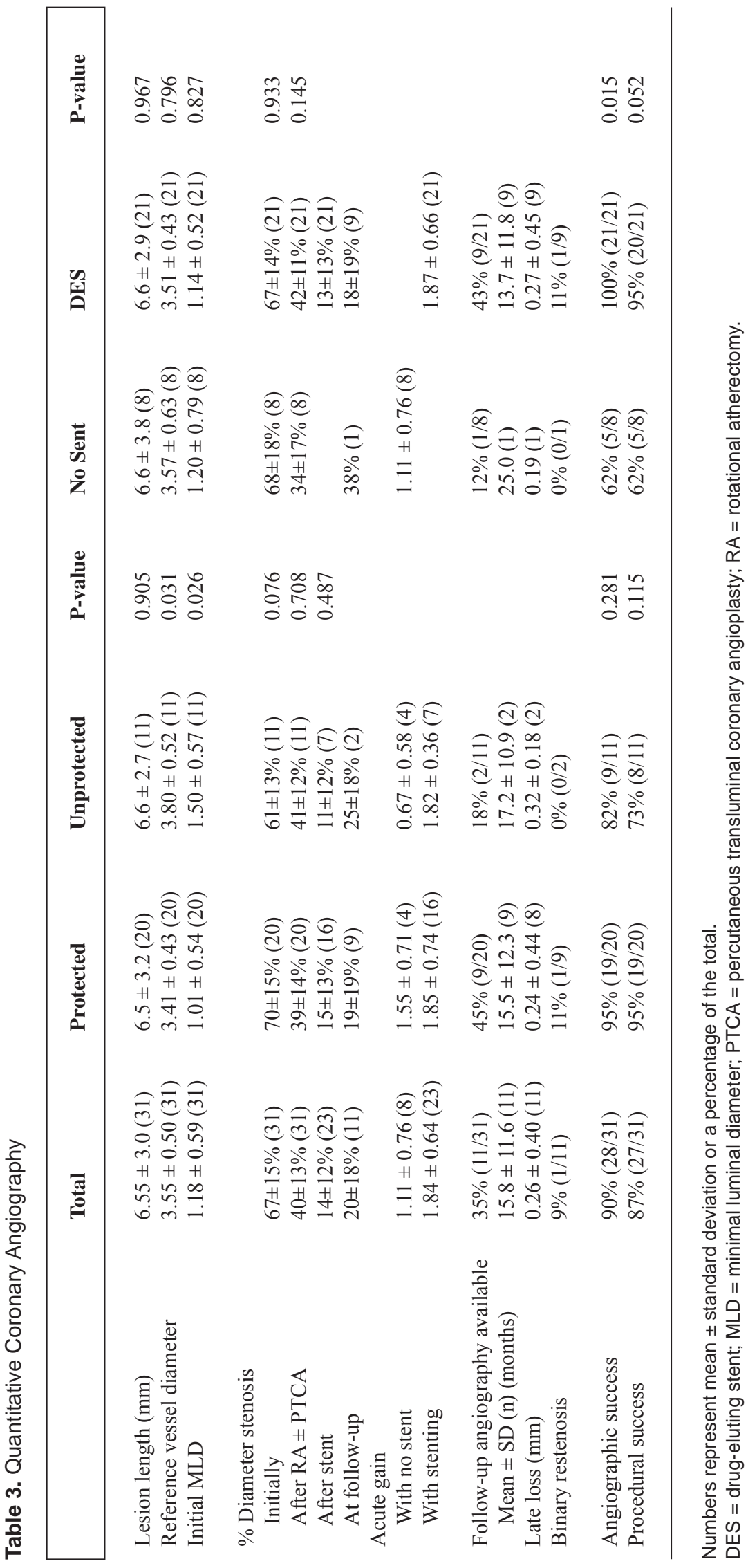


Table 4. In-Hospital Outcomes and Procedural Complications

\begin{tabular}{ll}
\hline & Total \\
\hline Death, \%(n)* & $3 \%(1 / 31)$ \\
CABG, \%(n) & $0 \%(0 / 31)$ \\
Q-wave MI, \%(n) & $0 \%(0 / 31)$ \\
Non Q-wave MI, \%(n) & $0 \%(0 / 31)$ \\
MACE, \%(n) & $3 \%(1 / 31)$ \\
& \\
Dissection, \%(n) & $0 \%(0 / 31)$ \\
Perforation, \%(n) & $0 \%(0 / 31)$ \\
Spasm, \%(n) & $0 \%(0 / 31)$ \\
Thrombosis, \%(n) & $0 \%(0 / 31)$ \\
No reflow, \%(n) & $0 \%(0 / 31)$ \\
Side branch occlusion, \%(n) & $0 \%(0 / 31)$ \\
Hypotension and/or bradycardia, \%(n) & $0 \%(0 / 31)$ \\
\hline
\end{tabular}

Numbers represent a percentage of the total.

* = 1 death was unprotected LMCA treated with a drug-eluting stent; CABG = coronary artery bypass graft surgery; $\mathrm{MACE}=$ major adverse cardiovascular events; $\mathrm{MI}=$ myocardial infarction .

(100\% vs. $62 \%$; $\mathrm{P}=0.0153)$. All 3 patients without angiographic success (because of residual diameter stenoses of 42$53 \%$ ) had a contraindication to $\mathrm{CABG}$ surgery and a reason to avoid a stent.

There were no procedural complications (Table 4). Acute MACE occurred in 1 of 31 patients (3\%); one 97-year-old patient with an unprotected LMCA, dementia and multiple comorbidities died of pneumonia, renal failure and congestive heart failure 14 days after the procedure.

The results of QCA are shown in Table 3. Acute gain after RA \pm balloon angioplasty was $0.93 \pm 0.62 \mathrm{~mm}$. Total acute gain for the 23 patients with stent implantation was $1.84 \pm 0.64 \mathrm{~mm}$. A mean initial percent diameter stenosis of $67 \pm 14 \%$ was reduced to a mean of $13 \pm 13 \%$ after RA and DES implantation.

Clinical follow-up was available in $74 \%$ of all patients and in $86 \%$ of the DES patients at a mean of $15.3 \pm 14.7$ months (Table 5). Overall, MACE occurred in 6 patients $(26 \%)$ at $11.9 \pm 10.6$ months (1 non-ST elevation myocardial infarction, 2 target vessel revascularizations, 3 deaths). During follow-up, MACE tended to occur less frequently in patients with protected LMCAs vs. unprotected LMCAs (P $=0.0697$ by log-rank; Fig. 1). No differences were observed in MACE rates or death rates in patients treated with a DES compared with no stent.

Following repeat PCI, 2 of the 6 patients with mid-term MACE were later documented to be free from angina. As of the last known follow-up $(16.8 \pm 14.1$ months $)$, nineteen of 22 patients $(87 \%)$ were alive and free from angina. Patients were more likely to be alive and free from angina if they had a protected LMCA (94\% vs. 57\% with unprotected LMCA; $\mathrm{P}=0.0564)$ and if they received a DES (89\% vs. 50\% with no stent; $\mathrm{P}=0.0281)$. Angiographic follow-up was available in 9 of $21(43 \%)$ patients with DESs at a mean of $13.7 \pm 11.8$ months revealing a mean late loss of $0.27 \pm 0.45 \mathrm{~mm}$ and binary restenosis in 1 of $9(11 \%)$ (Table 3$)$.

\section{Discussion}

This study sought to describe the contemporary use of RA in the LMCA to facilitate DES placement. The main outcomes of this analysis suggest that RA in the LMCA is safe and feasible and facilitates DES implantation with high procedural success. Compared with RA alone in the LMCA, RA and DES implantation is associated with a higher angiographic success rate. Patients were more likely to be alive and free from angina at final follow-up if they had a protected LMCA and received a DES.

The benefits of RA have previously been described and include improving angiographic outcomes and facilitating stent implantation in heavily calcified lesions [7-10, 21, 22] and preserving the patency of side branches in bifurcation and ostial lesions $[12,13]$. In heavily calcified lesions, RA combined with a DES resulted in target lesion revascularization rates ranging from $2 \%$ to $10.6 \%$ at 6 months to 3 years of follow-up [14-18]. As these reports focused on non-LMCA PCI, we sought to extend these concepts to the LMCA. These concepts are particularly relevant to LMCA lesions which often become symptomatic at the later stages of coronary artery disease, frequently years after prior CABG surgery, involve the distal bifurcation over $60 \%$ of the time and frequently contain severe calcification $[2,4,5]$.

The results of this contemporary series of 31 patients with LMCA lesions treated with RA suggest that the previous findings of RA in the non-LMCAs translate to the 


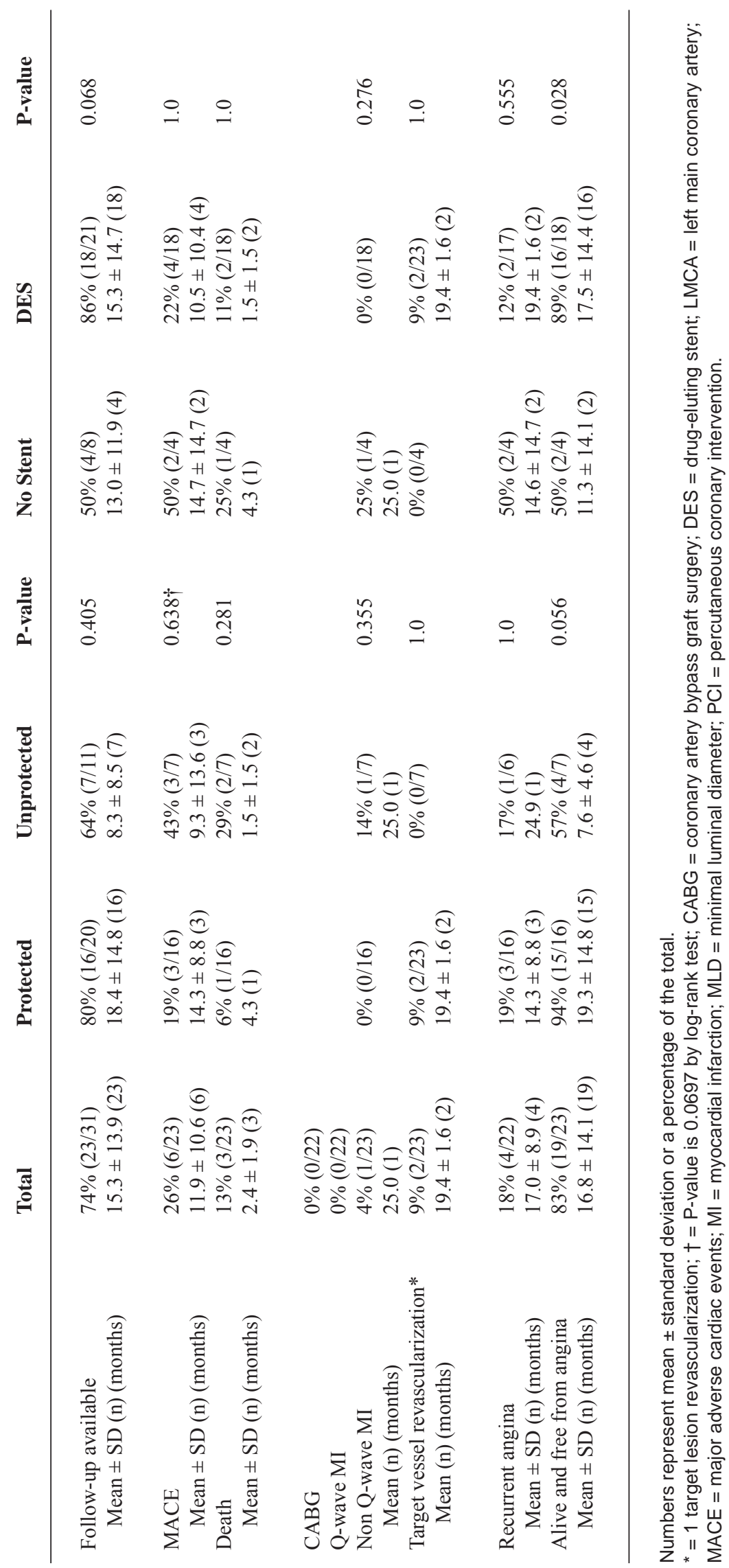




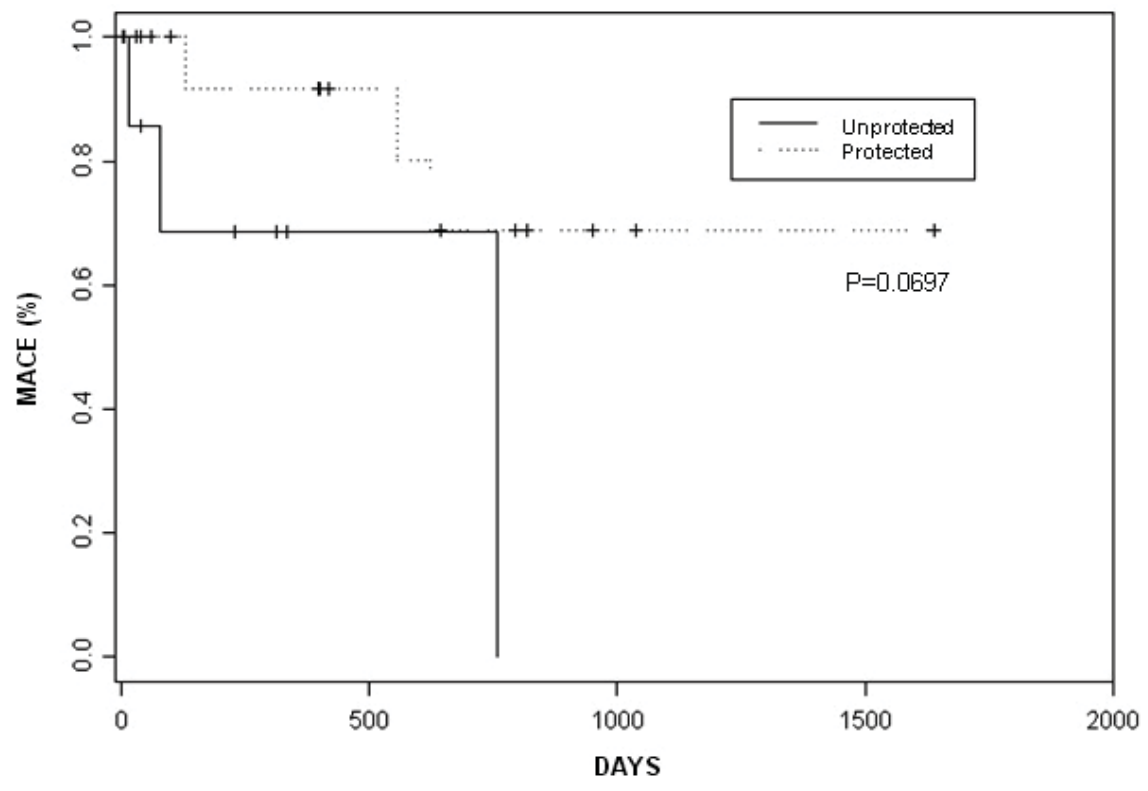

Figure 1. Major adverse cardiovascular events in patients with protected and unprotected left main coronary artery interventions. Kaplan-Meier incidence curves of major adverse cardiovascular events (MACE) in patients with protected and unprotected left main coronary artery interventions. Incidences in the 2 groups were statistically compared with a log-rank test.

LMCA. A DES was successfully implanted in all patients without a contraindication to DESs, despite severe vessel calcification. There were no procedural complications. One major adverse cardiovascular event $(3 \%)$ occurred prior to hospital discharge: a 97-year-old woman with an unprotected LMCA and multiple comorbidities died of pneumonia. The procedure was well tolerated by each patient and acute morbidity was minimal in this patient cohort with high-risk LMCA disease. Even in patients with unprotected LMCAs, RA was used in conjunction with left ventricular assist devices without procedural complications.

At last known follow-up (16.8 \pm 14.1 months), patients were more likely to be free from angina if they had a protected LMCA and received a DES. MACE tended to occur less frequently in patients with protected versus unprotected LMCAs. These results suggest that patients with protected LMCAs have favorable mid-term outcomes following RA with a DES. Patients with unprotected LMCAs can safely undergo RA, but data on mid-term outcomes is inconclusive.

This series is compatible with other reports on patients undergoing LMCA interventions. Death and MACE are known to occur more frequently in patients undergoing PCI for unprotected LMCA disease compared with protected LMCA disease [23]. Patients undergoing LMCA PCI have better long-term outcomes with DES implantation compared with no stent or even with bare metal stents [2]. Improved long-term outcomes in patients treated with DESs compared with no stent were not observed in our series, probably due to a small sample size and limited patient follow-up. Death and MACE rates (at about 15 months) were slightly higher for this entire cohort (death 13\%, MACE 26\%), DES group $(11 \%, 22 \%)$, and unprotected LMCA group (29\%, 43\%) compared with other reports of 12 month outcomes for patients with LMCA disease undergoing DES implantation ( $\sim 5 \%$, $\sim 10-15 \%)$ or CABG $(\sim 10 \%, \sim 23 \%)$ (beyond 12 months outcomes favor CABG compared with DES) [2]. The increase in death and MACE at 1 year observed in this series may be due to small sample size, incomplete patient follow-up, and most importantly selection bias. Unlike many reports with inclusion and exclusion criteria that exclude the most critically ill patients, this series, by selecting only patients for which RA was deemed necessary, selected only patients with severe coronary artery disease (severe calcification) and comorbid conditions (including myocardial infarction presentation and 1 patient in cardiogenic shock).

Initial reports of RA described relatively high complication rates including vessel dissection in the range of 5-10\% and perforation necessitating emergent $\mathrm{CABG}$ surgery in approximately $1 \%$ [22, 24-26)]. However, when RA is employed as a stand alone procedure, as in the initial reports, a relatively large burr-to-vessel ratio $(>0.7)$ was used to achieve optimal rotablation [24-26]. When RA is employed to facilitate stent implantation, smaller burr-to-vessel ratios are commonly used, ranging from 0.5 - 0.6 [9, 11, 15, 27]. In this cohort of LMCA lesions a final burr-to-vessel ratio of 0.43 was used. When RA is employed to facilitate stent expansion, optimal rotablation is not necessary, but rather the goal is to change the compliance of the calcified vessel so that optimal stent expansion can be achieved. By reducing the rigidity and eccentricity of a heavily calcified lesion, 
stent expansion is optimized and more symmetric [10]. As the LMCA exhibits substantial elastic recoil [19], balloon angioplasty does not contribute significantly to acute gain in the LMCA; most of the initial acute gain can be attributed to RA rather than balloon angioplasty. The benefit of RA, however, cannot be measured by its immediate acute gain, but rather by the final acute gain after stenting which could not be realized without adjuvant RA. Similarly, relatively smaller burr-to-vessel ratios are needed to debulk plaque burden and prevent side branch occlusion [13]. Adjunctive rotablation may have additional benefits when used with DESs by limiting trauma to the stent coating and improving drug delivery to the subintimal tissue [15].

The findings in the present report support the utility of RA in which a smaller burr-to-vessel ratio is used for heavily calcified lesions of the LMCA. The goal of RA in this setting is to facilitate stent implantation and to prevent occlusion of the left anterior descending and left circumflex coronary arteries when the distal bifurcation is involved. RA was used successfully as a "bail-out" procedure after the balloon catheter could not be delivered to a protected LMCA lesion in 2 patients. The use of RA probably spared these 2 patients from a repeat $\mathrm{CABG}$ surgery.

Left ventricular assist devices (mainly TandemHeart) were used in patients with depressed left ventricular function undergoing PCI on an unprotected LMCA lesion. The American College of Cardiology/American Heart Association guidelines recommend cardiac assist devices in such high-risk interventions [3].

The relatively small initial percent diameter stenosis is related to our use of the proximal LMCA to define reference vessel diameter, while most lesions involved the distal segment. Also, several lesions spanned both the LMCA and one of its branches with the true MLD distal to the bifurcation of the LMCA. For this report on LMCA interventions, QCA measurements were confined to the LMCA.

There are several limitations of the present study. As a retrospective analysis at a single center it is uncertain whether these results will translate prospectively to clinical practice in the community. This analysis included a small number of patients; they may not be representative of larger patient populations. Follow-up data was only available for patients who returned to our hospital to be evaluated by our physicians (23 of 31 patients; 74\%), and was limited to only a few months for several patients. The majority of patients with limited or no follow-up were referred from their primary cardiologist for a complex PCI, then returned to their primary cardiologist for follow-up care. Some late major adverse cardiac events may have been missed.

\section{Conclusion}

In summary, RA of the LMCA is safe and feasible and facilitates DES implantation with high procedural success. Favor- able mid-term outcomes were observed following RA and DES implantation in heavily calcified, protected LMCAs. The results suggest that routine use of RA prior to DES implantation may be considered in patients with heavily calcified lesions involving the distal LMCA.

\section{Acknowledgements}

Sharon Hale and John Pezzullo, your contributions to the statistical analysis are greatly appreciated.

\section{Conflict of Interest}

An educational grant from Boston Scientific was used to support this project. There are no other conflicts of interest.

\section{References}

1. Capodanno D, Di Salvo ME, Cincotta G, Miano M, Tamburino C. Usefulness of the SYNTAX score for predicting clinical outcome after percutaneous coronary intervention of unprotected left main coronary artery disease. Circ Cardiovasc Interv. 2009;2(4):302-308.

2. Kandzari DE, Colombo A, Park SJ, Tommaso CL, Ellis SG, Guzman LA, Teirstein PS, et al. Revascularization for unprotected left main disease: evolution of the evidence basis to redefine treatment standards. J Am Coll Cardiol. 2009;54(17):1576-1588.

3. Smith SC, Jr., Feldman TE, Hirshfeld JW, Jr., Jacobs AK, Kern MJ, King SB, III, Morrison DA, et al. ACC/ AHA/SCAI 2005 guideline update for percutaneous coronary intervention: a report of the American College of Cardiology/American Heart Association Task Force on Practice Guidelines (ACC/AHA/SCAI Writing Committee to Update the 2001 Guidelines for Percutaneous Coronary Intervention). J Am Coll Cardiol. 2006;47(1):e1-121.

4. Biondi-Zoccai GG, Lotrionte M, Moretti C, Meliga E, Agostoni P, Valgimigli M, Migliorini A, et al. A collaborative systematic review and meta-analysis on 1278 patients undergoing percutaneous drug-eluting stenting for unprotected left main coronary artery disease. Am Heart J. 2008;155(2):274-283.

5. Palmerini T, Sangiorgi D, Marzocchi A, Tamburino C, Sheiban I, Margheri M, Vecchi G, et al. Ostial and midshaft lesions vs. bifurcation lesions in 1111 patients with unprotected left main coronary artery stenosis treated with drug-eluting stents: results of the survey from the Italian Society of Invasive Cardiology. Eur Heart J. 2009;30(17):2087-2094.

6. Tran T, Brown M, Lasala J. An evidence-based approach 
to the use of rotational and directional coronary atherectomy in the era of drug-eluting stents: when does it make sense? Catheter Cardiovasc Interv. 2008;72(5):650-662.

7. Whitbourn RJ, Sethi R, Pomerantsev EV, Fitzgerald PJ. High-speed rotational atherectomy and coronary stenting: QCA and QCU analysis. Catheter Cardiovasc Interv. $2003 ; 60(2): 167-171$.

8. Hoffmann R, Mintz GS, Kent KM, Pichard AD, Satler LF, Popma JJ, Hong MK, et al. Comparative early and nine-month results of rotational atherectomy, stents, and the combination of both for calcified lesions in large coronary arteries. Am J Cardiol. 1998;81(5):552-557.

9. Henneke KH, Regar E, Konig A, Werner F, Klauss V, Metz J, Theisen K, et al. Impact of target lesion calcification on coronary stent expansion after rotational atherectomy. Am Heart J. 1999;137(1):93-99.

10. Hoffmann R, Mintz GS, Popma JJ, Satler LF, Kent KM, Pichard AD, Leon MB. Treatment of calcified coronary lesions with Palmaz-Schatz stents. An intravascular ultrasound study. Eur Heart J. 1998;19(8):1224-1231.

11. Schluter M, Cosgrave J, Tubler T, Melzi G, Colombo A, Schofer J. Rotational atherectomy to enable sirolimuseluting stent implantation in calcified, nondilatable de novo coronary artery lesions. Vascular Disease Management 2007:4(3):63-69.

12. Sharma SK, Bhalla N, Dangas G, Duvvuri S, Kakarala V, Marmur JD, Cocke TP, Ambrose JA. Rotational atherectomy prior to coronary stenting prevents side branch occlusion. J.Am.Coll.Cardiol. 1997:498A.

13. Tan RP, Kini A, Shalouh E, Marmur JD, Sharma SK. Optimal treatment of nonaorto ostial coronary lesions in large vessels: acute and long-term results. Catheter Cardiovasc Interv. 2001;54(3):283-288.

14. Rathore S, Matsuo H, Terashima M, Kinoshita Y, Kimura M, Tsuchikane E, Nasu K, et al. Rotational atherectomy for fibro-calcific coronary artery disease in drug eluting stent era: procedural outcomes and angiographic followup results. Catheter Cardiovasc Interv. 2010;75(6):919927.

15. Khattab AA, Otto A, Hochadel M, Toelg R, Geist V, Richardt G. Drug-eluting stents versus bare metal stents following rotational atherectomy for heavily calcified coronary lesions: late angiographic and clinical followup results. J Interv Cardiol. 2007;20(2):100-106.

16. Vaquerizo B, Serra A, Miranda F, Triano JL, Sierra G, Delgado G, Puentes A, et al. Aggressive plaque modification with rotational atherectomy and/or cutting balloon before drug-eluting stent implantation for the treatment of calcified coronary lesions. J Interv Cardiol. 2010;23(3):240-248.

17. Mezilis N, Dardas P, Ninios V, Tsikaderis D. Rotablation in the drug eluting era: immediate and long-term results from a single center experience. J Interv Cardiol. 2010;23(3):249-253.

18. Clavijo LC, Steinberg DH, Torguson R, Kuchulakanti PK, Chu WW, Fournadjiev J, Satler LF, et al. Sirolimus-eluting stents and calcified coronary lesions: clinical outcomes of patients treated with and without rotational atherectomy. Catheter Cardiovasc Interv. 2006;68(6):873-878.

19. Nayak AK, Davis R, Reddy HK, Krishnan MS, Voelker DJ, Aggarwal K. Left main coronary artery rotational atherectomy and stenting. South Med J. 2000;93(4):415423.

20. Kini AS. Coronary angiography, lesion classification and severity assessment. Cardiol Clin. 2006;24(2):153162, v.

21. Kiesz RS, Rozek MM, Ebersole DG, Mego DM, Chang CW, Chilton RL. Novel approach to rotational atherectomy results in low restenosis rates in long, calcified lesions: long-term results of the San Antonio Rotablator Study (SARS). Catheter Cardiovasc Interv. 1999;48(1):48-53.

22. MacIsaac AI, Bass TA, Buchbinder M, Cowley MJ, Leon MB, Warth DC, Whitlow PL. High speed rotational atherectomy: outcome in calcified and noncalcified coronary artery lesions. J Am Coll Cardiol. 1995;26(3):731-736.

23. Wood F, Bazemore E, Schneider JE, Jobe RL, Mann T. Technique of left main stenting is dependent on lesion location and distal branch protection. Catheter Cardiovasc Interv. 2005;65(4):499-503.

24. Mauri L, Reisman M, Buchbinder M, Popma JJ, Sharma SK, Cutlip DE, Ho KK, et al. Comparison of rotational atherectomy with conventional balloon angioplasty in the prevention of restenosis of small coronary arteries: results of the Dilatation vs Ablation Revascularization Trial Targeting Restenosis (DART). Am Heart J. 2003;145(5):847-854.

25. Whitlow PL, Bass TA, Kipperman RM, Sharaf BL, Ho KK, Cutlip DE, Zhang Y, et al. Results of the study to determine rotablator and transluminal angioplasty strategy (STRATAS). Am J Cardiol. 2001;87(6):699-705.

26. vom Dahl J, Dietz U, Haager PK, Silber S, Niccoli L, Buettner HJ, Schiele F, et al. Rotational atherectomy does not reduce recurrent in-stent restenosis: results of the angioplasty versus rotational atherectomy for treatment of diffuse in-stent restenosis trial (ARTIST). Circulation. 2002;105(5):583-588.

27. Moussa I, Di Mario C, Moses J, Reimers B, Di Francesco L, Martini G, Tobis J, et al. Coronary stenting after rotational atherectomy in calcified and complex lesions. Angiographic and clinical follow-up results. Circulation. 1997;96(1):128-136. 